\title{
Effect of low-addition soft contact lenses with decentered optical design on myopia progression in children: a pilot study
}

This article was published in the following Dove Press journal:

Clinical Ophthalmology

23 September 2014

Number of times this article has been viewed

\author{
Takashi Fujikado' \\ Sayuri Ninomiya ${ }^{2}$ \\ Takuma Kobayashi ${ }^{2}$ \\ Asaki Suzaki ${ }^{3}$ \\ Mitsuhiko $\mathrm{Nakada}^{3}$ \\ Kohji Nishida ${ }^{4}$
}

'Department of Applied Visual Science, Osaka University Graduate School of Medicine, Suita, ${ }^{2}$ Itami Chuo Eye Clinic, Itami, ${ }^{3}$ Menicon, Nagoya, ${ }^{4}$ Department of Ophthalmology, Osaka University Graduate School of Medicine, Suita, Japan
Correspondence: Takashi Fujikado Department of Applied Visual Science, Osaka University Graduate School of Medicine, 2-2 Yamadaoka, Suita, Osaka 565-087I, Japan

$\mathrm{Tel}+8 \mathrm{I} 66879345$ I

Fax +8I 668793458

Email fujikado@ophthal.med.osaka-u.ac.jp
Purpose: To investigate the effect of low-addition soft contact lenses (CLs) with decentered optical design on the progression of myopia in children in a pilot study.

Subjects and methods: Twenty-four Japanese children age 10-16 years with baseline myopia of -0.75 to $-3.50 \mathrm{D}$ sphere and $\leq 1.00 \mathrm{D}$ cylinder were studied. The new CLs were designed to have a nasal decentration with the optical center centered on the line of sight, and with progressive-addition power of $+0.5 \mathrm{D}$ peripherally. Monofocal soft CLs were used as controls. A pair of new CLs or control CLs was randomly assigned to the children, and they wore the lenses for 12 months during the first phase. Then, the type of CLs was changed, ie, a crossover design, and the children were observed for another 12 months during the second phase. The end points were changes in axial length and refractive error (spherical equivalent) under cycloplegia.

Results: The change of axial length in the new-CL and control-CL groups was not different between 12 months and baseline, the change of axial length between 12 months and 1 month in the new-CL group $(0.09 \pm 0.08 \mathrm{~mm})$ was significantly smaller $(47 \%)$ than that in the control-CL group $(0.17 \pm 0.08 \mathrm{~mm}, P<0.05)$. During the same period, the change of refractive error in the new-CL group was not significantly different from that in the control group. Neither the change in axial length nor refractive error in the new-CL group was significantly different from those in the control-CL group in the second phase.

Conclusion: This pilot study suggests that low-addition soft CLs with decentered optical design can reduce the degree of axial elongation in myopic children after an initial transient phase of CL wear. The reduction of the progression of myopia by low-addition soft CLs warrants further investigations.

Keywords: prevention of myopia, myopia progression, contact lens, progressive-addition lens

\section{Introduction}

Myopia in children and adolescents is principally caused by an elongation of the axial length after the emmetropization process. ${ }^{1}$ Once myopia develops, it can continue to progress throughout childhood. ${ }^{2}$ This is troubling, because pathological processes, such as retinal detachments, ${ }^{3}$ macular degeneration, ${ }^{4,5}$ and glaucoma, ${ }^{6-8}$ develop more frequently in eyes with higher myopia. Therefore, even partial prevention of the progression of myopia can provide important protection of the eye from the development of these pathological processes.

The strategies that have been used to prevent axial elongation are based on three concepts. The first concept is controlling the environmental conditions, such as increasing outdoor activities ${ }^{9,10}$ and reducing near visual tasks. ${ }^{11}$ The second concept 
is altering the optical properties of the eye, and the third concept is the use of pharmacological agents. ${ }^{12-14}$

For the second strategy, spectacles with progressiveaddition lenses (PALs) were developed based on the results of animal experiments showing that hyperopic defocus triggers an elongation of the axial length of the eye,${ }^{15}$ and clinical results showing that myopic eyes had a lag of accommodation. ${ }^{16}$ However, prospective randomized studies on PALs showed that the use of PALs had relatively small effects on the prevention of myopia progression. ${ }^{17-20}$

The most recent experiments on the effects of optical devices on refractive errors have been based on the possible role of relative peripheral hyperopia in the development of myopia. Experiments in animals have suggested that the peripheral retina can control eye growth in eyes without a fovea. ${ }^{21}$ The results of a 1-year trial of spectacle lenses designed to correct central vision and reduce or eliminate peripheral hyperopic defocus ${ }^{22}$ showed a significant reduction in the rate of myopia progression in a subgroup of younger children (6-12 years) with a parental history of myopia.

The use of spectacle lenses for the correction of peripheral refractive errors is limited, because of the changes in the fixation, ie, viewing objects through a peripheral part of the lens. The results of contact lenses (CLs) designed on the same principles as myopia-control spectacles showed a $34 \%$ reduction $(-0.57 \mathrm{D}$ with new CLs versus $-0.86 \mathrm{D}$ with spectacle lenses) in the progression of myopia and a $33 \%$ reduction in axial length $(0.27 \mathrm{~mm}$ with new CLs versus $0.40 \mathrm{~mm}$ with spectacles). ${ }^{23}$ These findings suggested the possibility that CLs designed to reduce the peripheral hyperopic blur may be useful in preventing the progression of myopia.

Recent studies have shown that overnight orthokeratology with CLs might also convert relative peripheral hyperopia to relative peripheral myopia, and could reduce the progression of myopia. ${ }^{24}$ Several investigative groups have reported that orthokeratology is effective in preventing axial elongation compared with monofocal soft CLs (SCLs) ${ }^{25}$ or spectacle lenses. ${ }^{26,27}$ However, the increase of higher-order aberration and the decrease of contrast sensitivity by orthokeratologic lenses ${ }^{28}$ might be a problem for children during the sensitive period of visual development.

We have developed new progressive-addition SCLs that have a decentered design to fit the optical center of the $\mathrm{CL}$ to the center of pupil. We used a decentered design, because the center of the pupil is shifted nasally from that of the cornea. However, high-addition multifocal CLs have been reported to have significantly larger spherical aberration compared with low-addition multifocal CLs, ${ }^{29}$ suggesting that the retinal image may be better with low-addition CLs. Therefore, we used additional-power CLs with lower power than high-addition progressive CLs, which are suitable for children undergoing visual development. We investigated whether these additional-power SCLs would reduce the progression of myopia in children in a randomized controlled study with a crossover design.

\section{Subjects and methods Study subjects}

The study was conducted from January 6, 2011 to March 29, 2013. The inclusion criteria were age $6-16$ years, children and parents agreed to wear randomly assigned CLs, spherical refractive error from $-0.75 \mathrm{D}$ to $-3.50 \mathrm{D}$ with astigmatism $<1.0 \mathrm{D}$, vision correctable to at least 20/20 in both eyes with spherical lenses, normal ocular findings, and willing to wear the lens type assigned to them and to adhere to the protocol schedule. The exclusion criteria were; anisometropia $\geq 1.0 \mathrm{D}$, presence of strabismus, ocular diseases other than refractive errors, and history of orthokeratologic lens, bifocal, or progressiveaddition spectacle wear in the past 12 months.

Twenty-four Japanese children who met the inclusion criteria were randomly assigned to either group A or B (Table 1). Randomization was done by a random-number table, and the outcome measurements were made by masked examiners. In group A, children wore low-addition progressive design CLs (new CLs) in the first phase. In group B, children wore CLs that had a monofocal design (control $\mathrm{CL}$ ) in the first phase. Group A consisted of three boys and eight girls, and their ages ranged from 12 to $16(14.3 \pm 1.3$, [mean \pm standard deviation]) years, and group B consisted of four boys and nine girls, and their ages ranged from 10 to $16(14.3 \pm 1.3)$ years.

The efficacy of myopic control of new CLs was determined by the differences in mean axial length changes in the two groups after 1 year. We sought $80 \%$ power to detect a $0.10 \mathrm{~mm}$ (standard deviation $0.07 \mathrm{~mm}$ ) difference in axial length elongation between the two groups with a significance level of 0.05 (two-tailed): the minimum number of subjects required in each group to complete the study was nine.

The study was conducted in accordance with the tenets of the Declaration of Helsinki, and the procedures were approved by the Ethics Committee of Osaka University. Informed consent was obtained in writing from all participants and their guardians after an explanation of the nature and possible consequences of the study. The trial was registered with the UMIN (University hospital Medical 
Table I Baseline data of children in the first phase

\begin{tabular}{llll}
\hline & New CLs & Control CLs & P-value \\
\hline Number of patient & $\mathrm{II}$ & 13 & - \\
Age & $14.3 \pm 1.3$ years & $13.1 \pm 1.9$ years & 0.1274 \\
& $(12-16$ eyes $)$ & $(10-16$ eyes $)$ & $0.7926^{*}$ \\
Male:female & $3: 8$ & $4: 9$ & 0.0963 \\
Corneal curvature & $7.86 \pm 0.24 \mathrm{~mm}$ & $7.81 \pm 0.17 \mathrm{~mm}$ & $0.386 \mathrm{I}$ \\
& $(7.34-8.26 \mathrm{~mm})$ & $24.97 \pm 0.62 \mathrm{~mm}$ & \\
Axial length & $24.74 \pm 0.73 \mathrm{~mm}$ & $(23.88-25.91 \mathrm{~mm})$ & 0.6666 \\
Spherical value & $(23.43-25.75 \mathrm{~mm})$ & $-2.64 \pm 0.99 \mathrm{D}$ & \\
& $-2.56 \pm 0.87 \mathrm{D}$ & $(-1.00--3.50 \mathrm{D})$ & \\
Cylindrical value & $(-0.75--3.25 \mathrm{D})$ & $-0.09 \pm 0.45 \mathrm{D}(\leq-1.00 \mathrm{D})$ & \\
\hline
\end{tabular}

Notes: *Chi-square test. All other values obtained using the Wilcoxon rank-sum test.

Abbreviation: CLs, contact lenses.

Information Network), and adhered to International Conference on Harmonisation and World Health Organization good clinical practice guidelines.

\section{Contact lens design}

The new CLs were made of a hydrogel lens material with an $8.6 \mathrm{~mm}$ base curve and $14.5 \mathrm{~mm}$ diameter (mipafilcon A; Menicon, Nagoya, Japan). The lenses had a central zone of $3.25 \mathrm{~mm}$ that corrected for the on-axis refractive error of the eye. Outside the central zone, the refractive power of the lenses increased progressively and positively to reach a relative positive power of $+0.50 \mathrm{D}$ at the edge of the peripheral treatment zone (Figures 1 and 2). The total treatment zone was $8 \mathrm{~mm}$. The new CLs were designed to have a decentered optical zone, with the center shifted $0.5 \mathrm{~mm}$ nasally from the geometric center of the lens. To prevent rotation of the lenses, the CLs had horizontal thick zones (Figure 1).

The control CLs were made of a hydrogel lens material with either an 8.5 or $9.0 \mathrm{~mm}$ base curve, or a $14.2 \mathrm{~mm}$ diameter (etafilcon A; Johnson \& Johnson, New Brunswick, NJ, USA). These lenses had a monofocal design, and the theoretical refractive power of the lenses increased negatively and progressively toward the periphery (Figure 2). The CLs were worn for at least 6 hours/day, 4 days/week, and were replaced on a daily basis.

\section{Study procedures}

The subjects were examined at baseline, after 1 month and 3 months, and then at 3-month intervals. After 12 months, subjects with the new CLs changed to the control CLs and those with control CLs changed to the new CLs, and both
A

Decentered optical zone

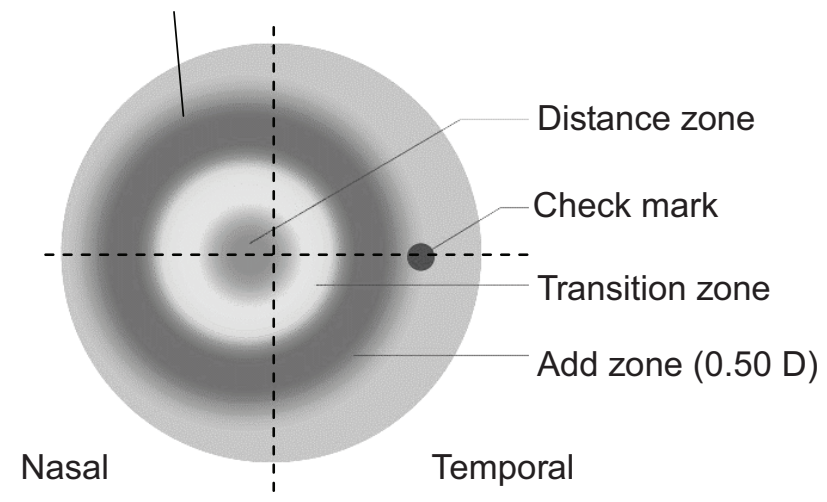

B

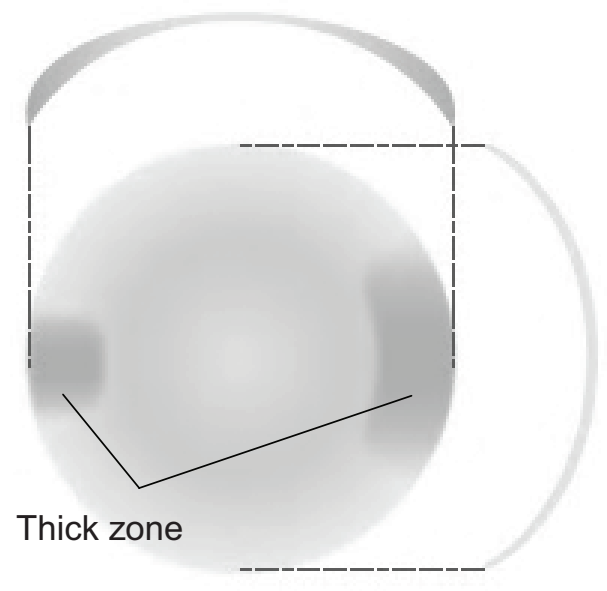

Figure I Design of low-addition soft contact lens (CL).

Notes: (A) Geometry of distance viewing and addition viewing zones. The CL was designed to have a decentered optical zone, with the center shifted $0.5 \mathrm{~mm}$ nasally from the geometric center of the lens. (B) The thick zones of the CL. To prevent CL rotation, it had horizontal thick zones. 


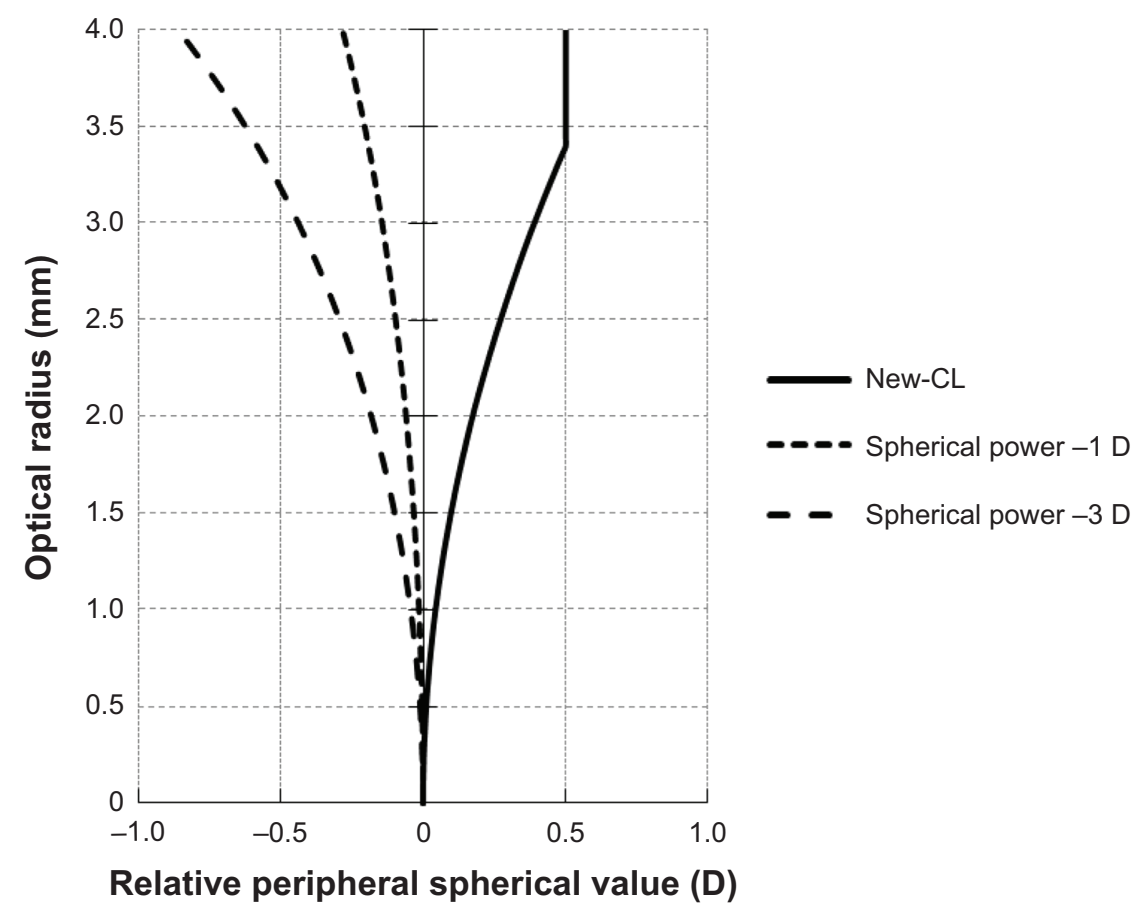

Figure 2 Simulated relative contact lens $(C L)$ power in the peripheral zone for the new-CL and the control-CL. In the new-CL, the theoretical refractive power of the lens increased progressively and positively to reach a relative positive power of $+0.50 \mathrm{D}$ at the edge of the peripheral zone. The control CLs were monofocal in design, and the theoretical relative refractive power of the lens increased negatively and progressively in the periphery. The short dashed line indicates the monofocal $\mathrm{CL}$ with spherical power of $-I D$. The long dashed line indicates monofocal $C L$ with spherical power of $-3 D$. The solid line indicates the new-CL.

groups were followed for another 12 months on the same examination schedule.

The primary outcome measures were changes in axial length measured at the 12- and 24-month visits and changes in central refractive error (spherical equivalent). Central refractive error was determined by cycloplegic autorefraction with an aberrometer (KR-9000PW; Topcon, Tokyo, Japan). Cycloplegia was induced with two drops of tropicamide $1 \%$, and measurements were taken at least 30 minutes later. Before the examination, the pupils were examined to ensure that they were dilated and were unresponsive to light.

Five autorefractor measurements were made for each eye and averaged. Axial length was measured by partial coherence interferometry (IOLMaster ${ }^{\circledR}$; Carl Zeiss Meditec, Jena, Germany). Three axial length measurements were made and averaged. Both cycloplegic autorefraction and axial length were measured at baseline, 1 month, and 3 months, and then at 3-month intervals. The measurements were taken by orthoptists masked to the type of CLs worn.

The peripheral refraction for each eye was measured using an open-field autorefractor (WAM-5500; Shigiya Machinery Works, Fukuyama, Japan) with and without the optical correction in place. Measurements were made in the nasal and temporal horizontal hemifields at $10^{\circ}, 20^{\circ}$, and $30^{\circ}$. Measurements at each fixation point were repeated five times. The average refractive error was calculated and analyzed statistically.

At baseline, the CLs were prescribed based on the refractive error of the cycloplegic manifest refraction, and were modified by a subjective refraction. At the follow-up visits, the child received new lenses only if their visual acuity had decreased to less than 20/20. To measure compliance, the subjects or guardians were asked the number of hours the lenses were worn on a given day, and the number of days on which the lenses were not worn.

\section{Statistical analyses}

The data for the children who attended at least the 12 -monthly visit were included in the analysis of the progression of myopia. In the second year, two children dropped out from the study because their families moved to another city.

Changes in axial length and spherical equivalent from baseline were calculated for each eye, and the values were compared between the two groups at 1,3,6,9, and 12 months. In the second year, data for ten children from the new-CL group and 12 children from the control-CL group were analyzed. Data of the right and left eyes were averaged. 
In one subject in whom only the right eye matched the inclusion criteria, data of this eye were used for statistical analysis. The Wilcoxon rank-sum test was used to compare the baseline characteristics between the two groups of subjects. Repeated-measures analysis of variance tests (and the Wilcoxon rank-sum test) were used to compare changes in axial length and refractive error during the study period. The level of statistical significance was set at $5 \%$. Data analyses were performed with the StatLight program (YUKMS, Tokyo, Japan).

\section{Results}

The baseline data of the subjects using the new CLs and the control CLs are compared in Table 1. At baseline, there were no significant differences in age, relative numbers of boys and girls, corneal curvature, axial length, or spherical and cylindrical refractive errors between the new-CL and control-CL groups.

\section{Relative peripheral refractive error profile}

Relative peripheral refractive error profiles in children who wore the new CLs, wore the control CLs, and did not wear any CLs, ie, naked eyes, were compared during the second year of the trial after the crossover (Figure 3). Relative peripheral refraction was not significantly different among the naked eyes, eyes with new CLs, and eyes with control CLs at any eccentricity.

\section{Axial length changes before and after crossover}

The change in axial length was significantly larger in the control-CL group compared with the new-CL group in phase 1 (repeated-measures ANOVA). The mean increase in axial length at 1 month was $0.05 \pm 0.02 \mathrm{~mm}$ for the new-CL group and $0.03 \pm 0.03 \mathrm{~mm}$ for the control-CL group. During this period, axial length elongated more in the new-CL group than in the control $\mathrm{CL}$ group $(P=0.03)$. At 3 months, the change in axial length was $0.05 \pm 0.04 \mathrm{~mm}$ in the new-CL group and $0.06 \pm 0.04$ $\mathrm{mm}$ in the control-CL group, and at 6 months the values were $0.08 \pm 0.04 \mathrm{~mm}$ in the new-CL group and $0.09 \pm 0.06 \mathrm{~mm}$ in the control-CL group. At 9 months, mean axial length was $0.10 \pm 0.04 \mathrm{~mm}$ in the new-CL group and $0.14 \pm 0.08 \mathrm{~mm}$ in the control-CL group. At 12 months, mean axial length was $0.15 \pm 0.07 \mathrm{~mm}$ in the new-CL group and $0.20 \pm 0.09 \mathrm{~mm}$ in the control-CL group. The differences in the increase in axial lengths at 3, 6,9, and 12 months were not significant (Figure 4).

When change in axial length was determined relative to that at 1 month, the change was significantly faster in the control group (repeated-measures analysis of variance). The change at 3 months was $0.03 \pm 0.01 \mathrm{~mm}$ for the new-CL group and $0.00 \pm 0.03 \mathrm{~mm}$ for the control-CL group, and at 9 months, values were $0.04 \pm 0.04 \mathrm{~mm}$ for the new-CL group and $0.11 \pm 0.06 \mathrm{~mm}$ for the control-CL group. At 12 months, values were $0.09 \pm 0.08 \mathrm{~mm}$ for the new-CL group and $0.17 \pm 0.08 \mathrm{~mm}$ for the control-CL group (Figure 5). The differences at these three times were statistically significant $(P<0.05)$.

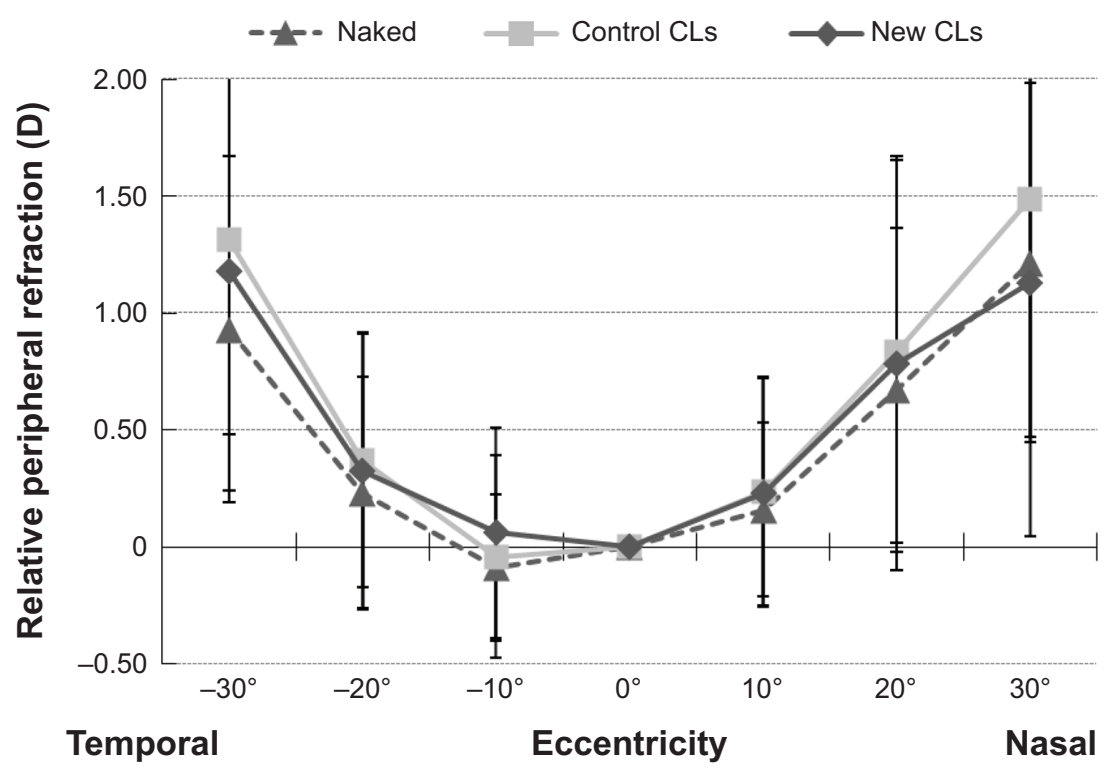

Figure 3 Relative peripheral refractive error profile with new contact lenses (CLs). The relative peripheral refractive error profiles in eyes wearing new CLs, wearing control CLs, and naked eyes were compared during the second year of the trial after the crossover. The relative peripheral refraction was not different (Steel-Dwass) among naked eyes, eyes with new CLs, and eyes with the control CLs at any eccentricity. 


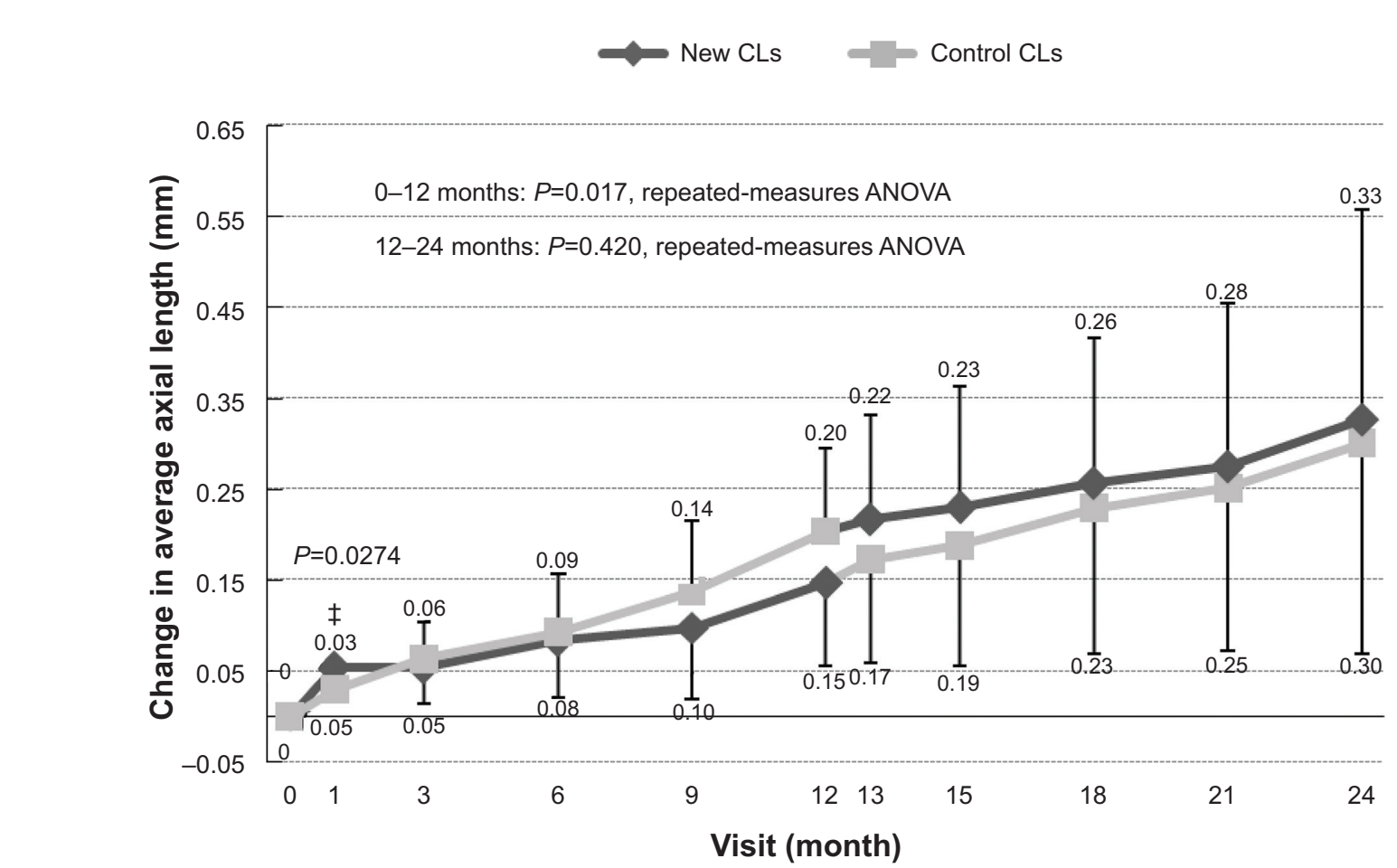

Figure 4 Mean changes in the axial length from baseline to 24 months. The differences were not significant except at I month when the axial length elongated more in the new-CL group than in the control-CL group.

Note: $: P<0.05$, Wilcoxon rank sum.

Abbreviations: CLs, contact lenses; ANOVA, analysis of variance.

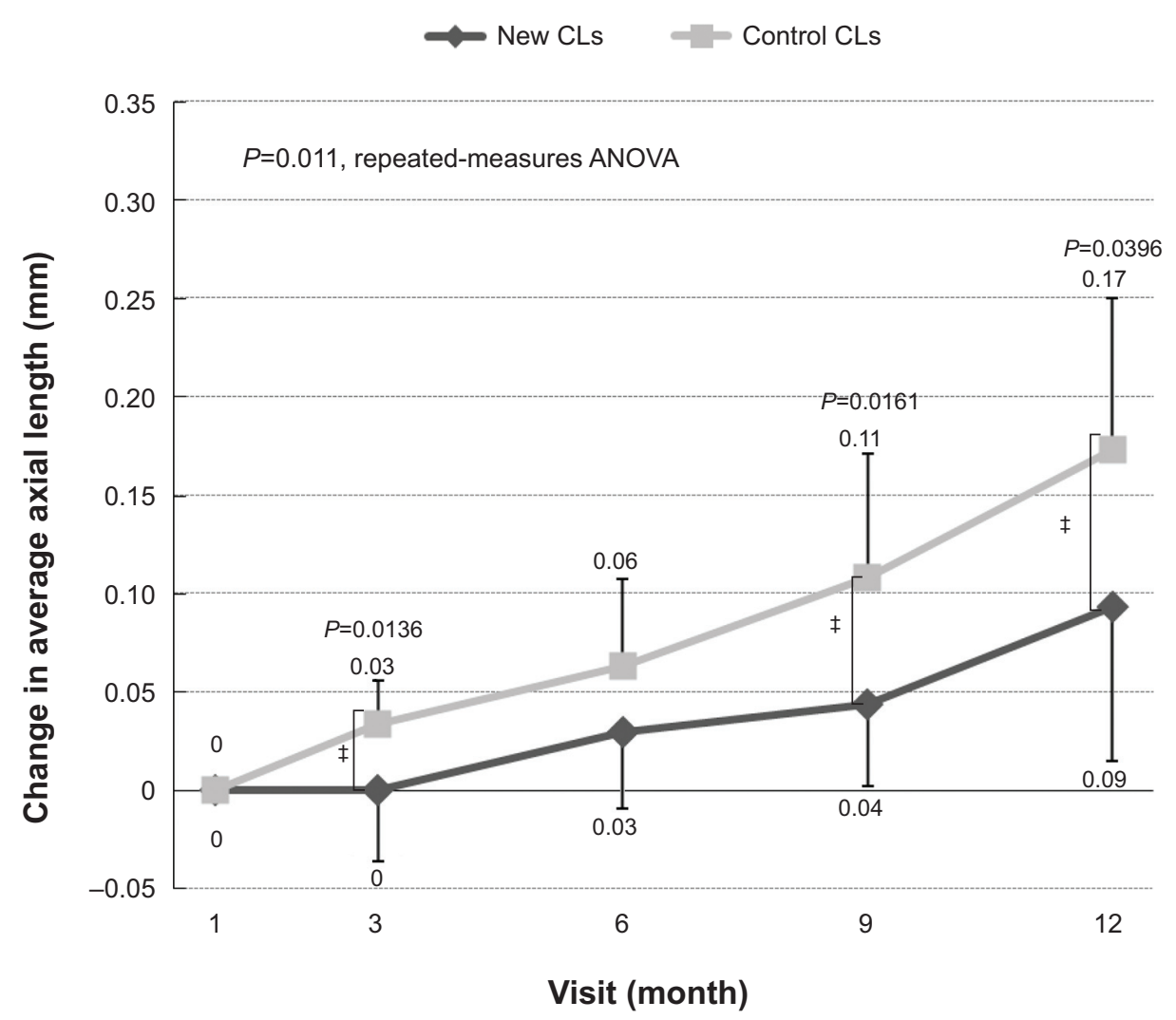

Figure 5 Mean changes in the axial length from I month to 12 months. The changes were significantly lower at 3,9 , and 12 months in the new- $C L$ group than in the control-CL group.

Note: ${ }^{\ddagger}<00.05$, Wilcoxon rank sum.

Abbreviations: CLs, contact lenses; ANOVA, analysis of variance. 
After the crossover, mean changes in axial length at 6 months were $0.06 \pm 0.08 \mathrm{~mm}$ for the new-CL group and $0.08 \pm 0.06 \mathrm{~mm}$ for the control-CL group. At 12 months, values were $0.12 \pm 0.16 \mathrm{~mm}$ and $0.15 \pm 0.11 \mathrm{~mm}$, respectively. The differences were not significant.

\section{Refractive error before and after crossover}

The mean change in refractive error at 1 month was $-0.47 \pm 0.41 \mathrm{D}$ for the new-CL group and $-0.12 \pm 0.36 \mathrm{D}$ for the control-CL group. During this period, refractive error became significantly more myopic in the new-CL group than in the control-CL group $(P=0.049)$. At 3 months, the mean change was $-0.49 \pm 0.35 \mathrm{D}$ for the new-CL group and $-0.21 \pm 0.30 \mathrm{D}$ for the control-CL group, and at 6 months, values were $-0.63 \pm 0.37 \mathrm{D}$ for the new-CL group and $-0.39 \pm 0.33 \mathrm{D}$ for the control-CL group. At 9 months, the mean refractive error was $-0.72 \pm 0.42 \mathrm{D}$ for the new-CL group and $-0.49 \pm 0.31 \mathrm{D}$ for the control-CL group, and at 12 months, the mean refractive error was $-0.84 \pm 0.42 \mathrm{D}$ for the new-CL group and $-0.62 \pm 0.43 \mathrm{D}$ for the control-CL group. These differences were not statistically significant (Figure 6).

When we measured the change in refractive error relative to that at 1 month, the change at 9 months was $-0.25 \pm 0.20 \mathrm{D}$ for the new-CL group and $-0.37 \pm 0.18 \mathrm{D}$ for the control-CL group, and at 12 months values were $-0.37 \pm 0.33 \mathrm{D}$ and $-0.50 \pm 0.18 \mathrm{D}$, respectively (Figure 7). These differences were not statistically significant.
After the crossover, the mean changes in refractive error at 6 months were $-0.18 \pm 0.28 \mathrm{D}$ for the new-CL group and $-0.30 \pm 0.27 \mathrm{D}$ for the control-CL group. At 12 months, values were $-0.29 \pm 0.34 \mathrm{D}$ and $-0.31 \pm 0.30 \mathrm{D}$, respectively. These differences were not statistically significant.

\section{Best-corrected visual acuity}

Best-corrected visual acuity (BCVA), measured in logarithm of the minimal angle of resolution (logMAR) units, was not significantly different between the new-CL group and the control-CL group at baseline $(-0.11 \pm 0.05 \log$ MAR units for the new-CL group and $-0.12 \pm 0.05 \log$ MAR units for the control-CL group, $P=0.36$ ). At the 6-month visit, BCVA was $-0.13 \pm 0.05 \log M A R$ units for the new-CL group and $-0.12 \pm 0.05 \log$ MAR units for the control-CL group ( $P=0.84$ ), and at 12 months, BCVA was $-0.14 \pm 0.04 \log$ MAR units for the new-CL group and $-0.15 \pm 0.04 \log$ MAR units for the control-CL group $(P=0.26)$. BCVA was not significantly different between the new-CL group and the control-CL group at 18 months after the crossover $(-0.15 \pm 0.03 \log$ MAR units for the new-CL group and $-0.16 \pm 0.03 \log$ MAR units for the control-CL group, $P=0.84$ ), and at the 24-month visit, BCVA was $-0.17 \pm 0.02 \log$ MAR units for the new-CL group and $-0.16 \pm 0.04 \log$ MAR units for the control group $(P=0.50)$.

\section{Compliance with lens wear}

The average days/week of wearing the CLs were $6.77 \pm 0.44$ days/week in the new-CL group compared with

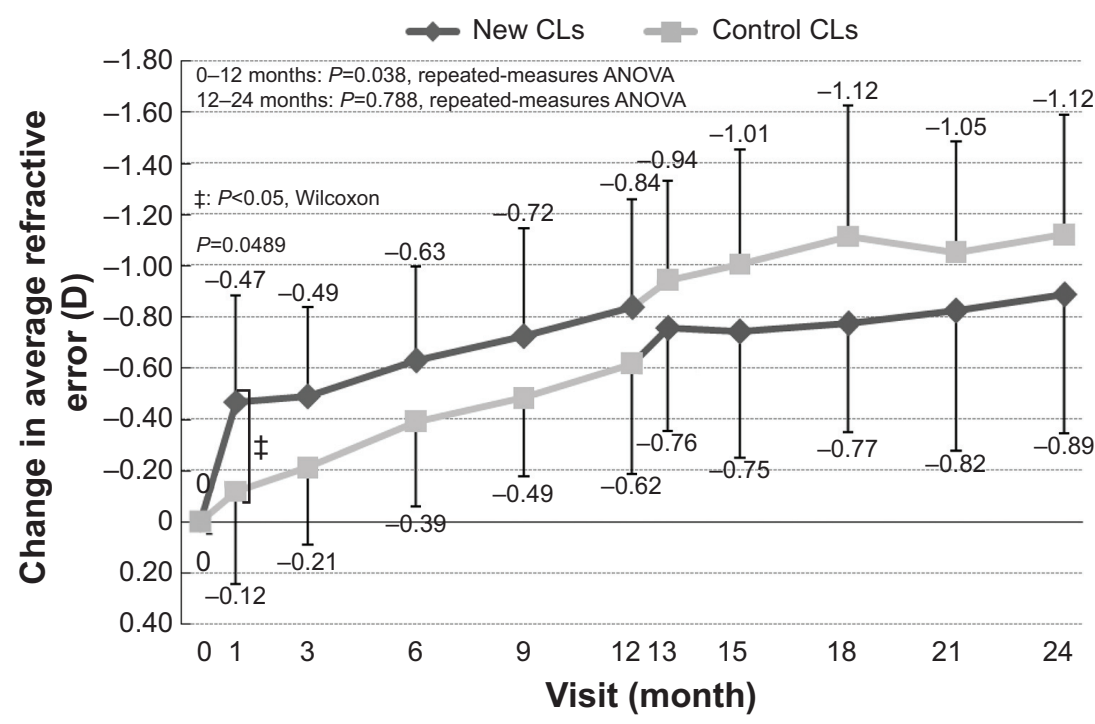

Figure 6 Mean changes in the refractive error from baseline to 24 months. The differences were not significant, except at I month, where the refractive error increased more in the new-CL group than the control-CL group.

Abbreviations: CLs, contact lenses; ANOVA, analysis of variance. 


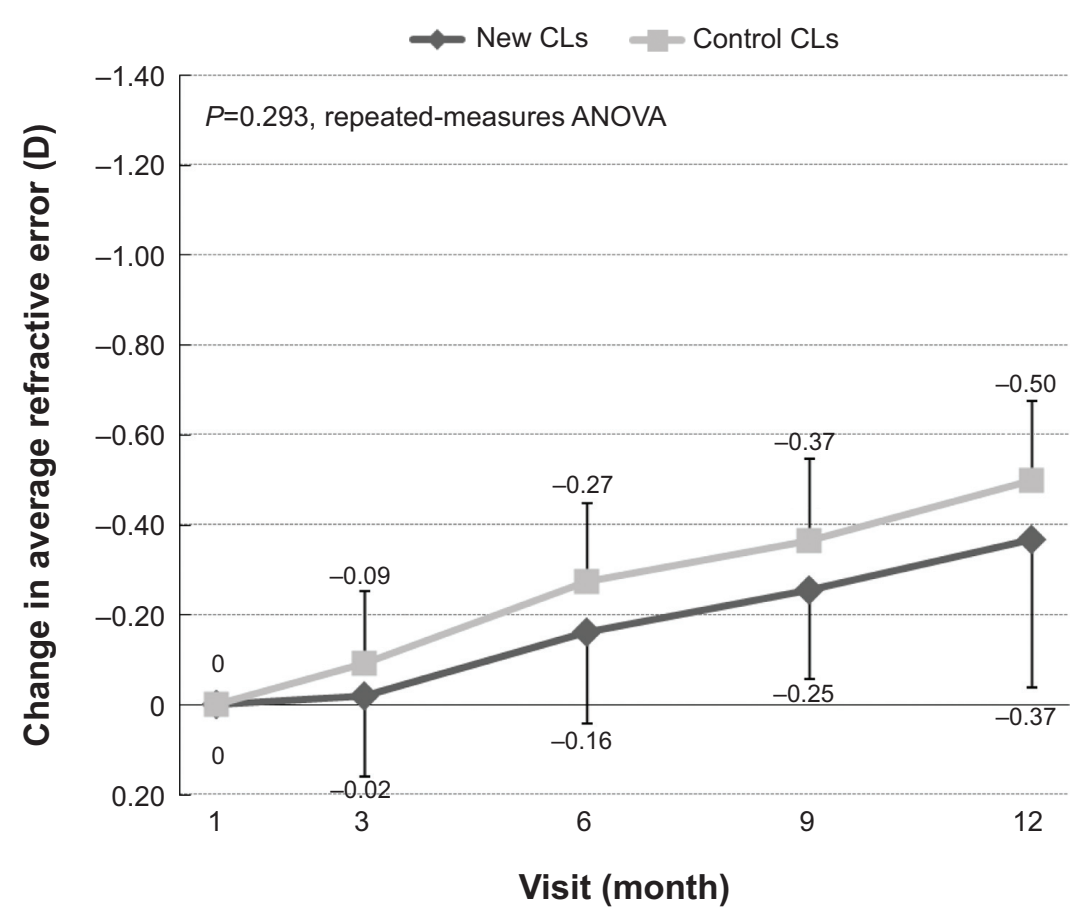

Figure 7 Mean changes in spherical equivalent refractive error from I month to 12 months. The changes in refractive error at I month were not significantly different between the new-CL group and the control-CL group.

Abbreviations: CLs, contact lenses; ANOVA, analysis of variance.

$6.72 \pm 0.55$ days/week in the control-CL group in the first phase $(P=0.83)$. In the second phase, children with the new CLs wore their CLs for 6.88 \pm 0.44 days/week compared with $6.70 \pm 0.47$ days/week for children wearing control CLs $(P=0.47)$.

The average time to wear CLs/day was $13.2 \pm 1.0$ hours/day in the new-CL group and 13.2 \pm 1.1 hours/day in the control-CL group $(P=1.00)$ in the first phase, and was $13.2 \pm 0.9$ hours/ day in the new-CL group and $13.6 \pm 0.8$ hours/day in the control-CL group $(P=0.35)$ in the second phase.

\section{Discussion}

We examined the effect of a low-addition progressive power lens with a decentered design on the progression of myopia in young children (Figures 1 and 2). Although the additional power was low $(+0.5 \mathrm{D})$, the difference of the relative lens power between new CLs and monofocal CLs (central power of $-3.0 \mathrm{D}$ ) at $3 \mathrm{~mm}$ periphery was about $0.9 \mathrm{D}$ theoretically (Figure 2). This should reduce the peripheral refractive error of myopic eyes when the new CLs are worn.

In the eyes of myopic children, relative refractive error at the periphery was not significantly reduced by wearing the new CLs compared to monofocal CLs (Figure 3). Sankaridurg et al demonstrated a significant reduction in peripheral hyperopic defocus using progressive-addition CLs with maximum additional power of $2.0 \mathrm{D}$ in myopic children. ${ }^{23}$
The discrepancy may have been caused by the difference in the additional power of the lens.

The new CLs were designed to have a decentered optical zone, with the center shifted $0.5 \mathrm{~mm}$ nasally from the geometric center of the lens. The value of the decentration was determined from the difference between the geometrical center of the CLs and the center of the entrance pupil in Japanese adult subjects. ${ }^{30}$ To prevent a rotation of the lens, the lens had a horizontal thick zone. This design is theoretically effective for the prevention of myopic shift. Indeed, Sankaridurg et $\mathrm{al}^{23}$ demonstrated that nasal-temporal asymmetry in peripheral refractive error changed when myopic children wore concentric and radially symmetrical CLs. They explained that this asymmetry could be caused by the systematic decentering of the CLs from the visual axis.

With our new CLs, there was a relatively small nasaltemporal asymmetry in peripheral refractive error (Figure 3). Therefore, the new CLs have an advantage over concentric and radially symmetrical CLs for the focus of objects on the peripheral retina symmetrically.

During the 11-month first-phase period after the initial 1-month trial, axial elongation was significantly reduced in the group wearing the new CLs compared to that of the control-CL group at 3, 9, and 12 months (Figure 5). However, during the first month of the first phase, axial length significantly increased in the group wearing the new CLs compared 
to the group wearing the control CLs (Figure 4). These data suggest that after an initial transient stage, the new CLs are effective in reducing axial elongation in myopic eyes. The reason for the initial axial elongation in the new CLs is not clear in this pilot study, and should be clarified in the next study with a larger number of subjects.

The rate of reduction of axial length elongation with the low-addition SCLs (47\%) was comparable to that reported by Sankaridurg et al where they used SCLs with additional power of +2.0 D. ${ }^{23}$ Ocular higher-order aberrations are reported to be greater with high-addition CLs compared with low-addition CLs. ${ }^{29}$ Therefore, it may be better to wear low-addition SCLs for children undergoing visual development if the reduction rate in axial length elongation is comparable.

Refractive error was not significantly different between the new-CL group and the control-CL group during the 11 months of the first phase after the initial 1-month trial (Figure 7). However, axial elongation was significantly reduced in the new-CL group. This discrepancy may have been caused by the small sample size.

In the second phase after the crossover, neither change in axial length nor refractive error in the new-CL group was significantly different from that in the control-CL group. An initial increase in axial length in the new-CL group was not observed in the second phase (Figure 4). These findings are similar to clinical trials with crossover design, which sometimes show similar results. For example, in an Okayama study (crossover design) using progressive-addition spectacles for children, PALs were effective in preventing a progression of myopia in the first year but not effective in the second year after the crossover. ${ }^{20}$ Two possibilities exist for these discrepancies. One was the age of wearing the new CLs; patients were delayed 1 year to start using the new CLs in the second-year study group. A second possibility was a carryover effect, where the experience of the first-year trial affected the second-year results.

In the eyes that wore the low-addition CLs, hyperopic blur in the peripheral retina was not significantly reduced compared with the control CLs; however, a suppression of axial elongation was observed after an initial transient phase. The reason for the reduction in axial elongation is not clear, but reduced accommodation for near targets by low-addition CLs could be a factor. Further investigations are necessary on this point.

The limitations of this study were the small number of patients and the short follow-up period. A larger number of patients with a longer follow-up period are needed to determine the effectiveness of low-addition SCLs with decentered optical design on myopia progression in children.

\section{Conclusion}

This pilot study suggests that low-addition SCLs with decentered optical design might reduce axial elongation in myopic children after an initial transient phase of CL wear. Larger numbers of patients and longer follow-up periods are necessary using this lens design to ensure the longevity of these effects on myopia progression.

\section{Disclosure}

$\mathrm{AS}$ and $\mathrm{MN}$ are employees of Menicon. The authors report no other conflicts of interest in this work.

\section{References}

1. Mutti DO, Mitchell GL, Sinnott LT, et al. Corneal and crystalline lens dimensions before and after myopia onset. Optom Vis Sci. 2012 $89: 251-262$

2. Tokoro K, Suzuki K. Changes in ocular refractive components and development of myopia during seven years. Jpn J Ophthalmol. 1969; 13:27-34.

3. Sheu SJ, Ger LP, Ho WL. Late increased risk of retinal detachment after cataract extraction. Am J Ophthalmol. 2010;149:113-119.

4. Iwase A, Araie M, Tomidokoro A, Yamamoto T, Shimizu H, Kitazawa Y. Prevalence and causes of low vision and blindness in a Japanese adult population: the Tajimi Study. Ophthalmology. 2006;113:1354-1362.

5. Morgan IG, Ohno-Matsui K, Saw SM. Myopia. Lancet. 2012;379: 1739-1748.

6. Wong TY, Klein BE, Klein R, Knudtson M, Lee KE. Refractive errors, intraocular pressure, and glaucoma in a white population. Ophthalmology. 2003;110:211-217.

7. Suzuki Y, Iwase A, Araie M, et al. Risk factors for open-angle glaucoma in a Japanese population: the Tajimi Study. Ophthalmology. 2006;113: $1613-1617$.

8. Xu L, Wang Y, Wang S, Jonas JB. High myopia and glaucoma susceptibility: the Beijing Eye Study. Ophthalmology. 2007;114:216-220.

9. Sherwin JC, Reacher MH, Keogh RH, Khawaja AP, Mackey DA, Foster PJ. The association between time spent outdoors and myopia in children and adolescents: a systematic review and meta-analysis. Ophthalmology. 2012;119:2141-2151.

10. Guo Y, Liu LJ, Xu L, et al. Outdoor activity and myopia among primary students in rural and urban regions of Beijing. Ophthalmology. 2013; 120:277-283.

11. Ip JM, Saw SM, Rose KA, et al. Role of near work in myopia: findings in a sample of Australian school children. Invest Ophthalmol Vis Sci. 2008;49:2903-2910.

12. Chia A, Chua WH, Cheung YB, et al. Atropine for the treatment of childhood myopia: safety and efficacy of $0.5 \%, 0.1 \%$, and $0.01 \%$ doses (Atropine for the Treatment of Myopia 2). Ophthalmology. 2012;119: 347-354.

13. Siatkowski RM, Cotter SA, Crockett RS, Miller JM, Novack GD, Zadnik K. Two-year multicenter, randomized, double-masked, placebocontrolled, parallel safety and efficacy study of $2 \%$ pirenzepine ophthalmic gel in children with myopia. J AAPOS. 2008;12:332-339.

14. Tan DT, Lam DS, Chua WH, Shu-Ping DF, Crockett RS. One-year multicenter, double-masked, placebo-controlled, parallel safety and efficacy study of $2 \%$ pirenzepine ophthalmic gel in children with myopia. Ophthalmology. 2005;112:84-91. 
15. Smith EL 3rd, Hung LF. The role of optical defocus in regulating refractive development in infant monkeys. Vision Res. 1999;39:1415-1435.

16. Gwiazda J, Thorn F, Bauer J, Held R. Myopic children show insufficient accommodative response to blur. Invest Ophthalmol Vis Sci. 1993;34: 690-694.

17. Leung JT, Brown B. Progression of myopia in Hong Kong Chinese schoolchildren is slowed by wearing progressive lenses. Optom Vis Sci. 1999;76:346-354.

18. Edwards MH, Li RW, Lam CS, Lew JK, Yu BS. The Hong Kong progressive lens myopia control study: study design and main findings. Invest Ophthalmol Vis Sci. 2002;43:2852-2858.

19. Gwiazda J, Hyman L, Hussein M, et al. A randomized clinical trial of progressive addition lenses versus single vision lenses on the progression of myopia in children. Invest Ophthalmol Vis Sci. 2003;44: $1492-1500$.

20. Hasebe S, Ohtsuki H, Nonaka T, et al. Effect of progressive addition lenses on myopia progression in Japanese children: a prospective, randomized, double-masked, crossover trial. Invest Ophthalmol Vis Sci. 2008;49:2781-2789.

21. Smith EL 3rd, Kee CS, Ramamirtham R, Qiao-Grider Y, Hung LF. Peripheral vision can influence eye growth and refractive development in infant monkeys. Invest Ophthalmol Vis Sci. 2005;46:3965-3972.

22. Sankaridurg P, Donovan L, Varnas S, et al. Spectacle lenses designed to reduce progression of myopia: 12-month results. Optom Vis Sci. 2010; 87:631-641.

23. Sankaridurg P, Holden B, Smith E 3rd, et al. Decrease in rate of myopia progression with a contact lens designed to reduce relative peripheral hyperopia: one-year results. Invest Ophthalmol Vis Sci. 2011;52: 9362-9367.
24. Charman WN, Mountford J, Atchison DA, Markwell EL. Peripheral refraction in orthokeratology patients. Optom Vis Sci. 2006;83: 641-648.

25. Walline JJ, Jones LA, Sinnott LT. Corneal reshaping and myopia progression. Br J Ophthalmol. 2009;93:1181-1185.

26. Hiraoka T, Kakita T, Okamoto F, Takahashi H, Oshika T. Long-term effect of overnight orthokeratology on axial length elongation in childhood myopia: a 5-year follow-up study. Invest Ophthalmol Vis Sci. 2012;53:3913-3919.

27. Cho P, Cheung SW. Retardation of myopia in orthokeratology (ROMIO) study: a 2-year randomized clinical trial. Invest Ophthalmol Vis Sci. 2012;53:7077-7085.

28. Hiraoka T, Okamoto C, Ishii Y, Kakita T, Okamoto F, Oshika T. Time course of changes in ocular higher-order aberrations and contrast sensitivity after overnight orthokeratology. Invest Ophthalmol Vis Sci. 2008;49:4314-4320.

29. Gifford P, Cannon T, Lee C, Lee D, Lee HF, Swarbrick HA. Ocular aberrations and visual function with multifocal versus single vision soft contact lenses. Cont Lens Anterior Eye. 2013;36:66-73.

30. Tanaka H, Sawano T, Oyama H, Hibino S. [Evaluation of optical decentered bifocal contact lens]. J Jpn Contact Lens Soc. 1995;37:158-161. Japanese.
Clinical Ophthalmology

\section{Publish your work in this journal}

Clinical Ophthalmology is an international, peer-reviewed journal covering all subspecialties within ophthalmology. Key topics include: Optometry; Visual science; Pharmacology and drug therapy in eye diseases; Basic Sciences; Primary and Secondary eye care; Patient Safety and Quality of Care Improvements. This journal is indexed on Submit your manuscript here: http://www.dovepress.com/clinical-ophthalmology-journal

\section{Dovepress}

PubMed Central and CAS, and is the official journal of The Society of Clinical Ophthalmology (SCO). The manuscript management system is completely online and includes a very quick and fair peer-review system, which is all easy to use. Visit http://www.dovepress.com/ testimonials.php to read real quotes from published authors. 